\title{
Shear stress regulates eNOS signaling in human umbilical vein endothelial cells via SRB1-PI3K- APl pathway
}

\begin{abstract}
Background: The occurrence and development of atherosclerosis are closely related to shear stress on the endothelial cells. However, related molecular mechanisms of controlling mechanical signal transduction in endothelial cells remain largely unknown.
\end{abstract}

Aim of the Study: To obtain this goal, here, a parallel-plate flow chamber was used to impose laminar shear stress (8.4 dyne $/ \mathrm{cm}^{2}$ ) on endothelial cells for 0 minute ( $\left.\mathrm{min}\right), 10 \mathrm{~min}, 30 \mathrm{~min}, 60 \mathrm{~min}$, and $120 \mathrm{~min}$.

Subjects and Methods: The RNA interference was employed to down-regulate scavenger receptor class B1 (SRB1) and LY294002 was performed to block the phosphorylation status of the phosphoinositide 3-kinase pathway. Then, the cell status was observed under the microscope, followed by detection of phosphorylated phosphoinositide 3-kinase (PI3K), p38, extracellular signal-regulated protein kinases 1 and 2 and the binding activities of activator protein 1 , nuclear factor$\mathrm{KB}$ and endothelial nitric oxide synthase (eNOs) by western blot. The results showed that shear stress $\left(8.4 \mathrm{dyne} / \mathrm{cm}^{2}\right)$ for 60 min induced obvious cell morphologic changes. Meanwhile, PI3K phosphorylation and eNOS expression were significantly increased by shear stress while reversed by SRB1 si-RNA and LY294002. Additionally, shear stress caused the enhanced binding activity of nuclear transcription factor activator protein 1 (AP-1) to DNA from 10 to $120 \mathrm{~min}$, while only at 30 and 60 min for NF-KB. After treatment with SRB1 si-RNA and LY294002 inhibitor, the binding activities of AP-1 and DNA was significantly decreased, however, there was no effect on NF-KB binding activity.

Results: Therefore, SRB1 plays a crucial role in mediating shear stress-induced eNOS activation in endothelial cells via PI3K-AP1 pathway. These findings will provide an important understanding of shear stress-induced cell alterations in clinic detection.

Keywords: Shear stress, eNOS signaling, human umbilical vein endothelial cells, SRB1-PI3K-AP1 pathway, gene interference, atherosclerosis

\section{Introduction}

It has been demonstrated that hemodynamic forces influence vascular endothelium function [1]. In addition, there are numerous membrane receptors in endothelial cells that can sense and transmit mechanical signals into nuclear signals through mechanosensitive signaling pathways [2,3]. Increasing evidence in the clinical studies have shown that atherosclerosis (AS) occurs preferentially at arterial branches and curvatures, which are the portions of the vasculature exposed to disturbed flow and low shear stress [4-6]. Therefore, low shear stress plays important roles in the development of atherosclerosis. Thus, searching for the underlying molecular mechanisms may provide potential intervening and therapeutic strategies in AS.

It was showed that in the arteriosclerotic regions, the endothelium has a proinflammatory phenotype associated with low nitric oxide secretion, reduced barrier function, and increased pro-coagulant and pro-proliferative properties, which was closely associated with the activity of endothelial nitric oxide synthase (eNOS) [7]. In the past decade, several studies have examined mechanosensitive receptors, but exactly how shear stress affects AS genesis remains unclear [8]. Previous reports have demonstrated that scavenger receptor class B1 (SRB1) can couple with High-Density Lipoprotein (HDL) for stimulation and respond to mechanical signals [9]. In addition,

\section{Ying Zhang ${ }^{{ }^{*}}$, Ya-Hui Dong ${ }^{*}$, Bin Liao ${ }^{2,3}$, Yong-Mei $\mathrm{Nie}^{2}$, Ju-yi Wan ${ }^{2,3}$, Liu-Lin Xiong ${ }^{4}$, Yong $\mathrm{Fu}^{2}$, Xiao-Jun $\mathrm{Xie}^{2}$, and Feng-Xu Yu $\mathbf{u}^{2,3}$}

${ }^{1}$ Department of Anesthesiology, Traditional Chinese Medicine Hospital of Southwest Medical University, Luzhou, 646000, China ${ }^{2}$ Department of Cardiovascular Surgery of the Affiliated Hospital of Southwest Medical University, Luzhou, 646000, China

${ }^{3}$ Key Laboratory of Medical Electrophysiology, Ministry of Education of China, Luzhou, 646000, China ${ }^{4}$ Institute of Neurobiological Disease, Department of Anesthesiology, 
suppressing $\mathrm{HDL}$ concentration polarization might stimulate atherosclerosis development $[10,11]$. Additionally, in the arterial wall, SRB1 expressed by macrophages may also prevent AS by stimulating cholesterol efflux and inhibiting foam cell formation [12]. We, therefore, hypothesized that shear stress interferes with lipid traffic to affect AS by regulating SRB1 expression.

We previously demonstrated that SRB1 was involved in shear stress-regulated endothelial cell function [13]. To clarify the function of SRB1 and its underlying molecular mechanism in the endothelial cells subjected to shear stress, we examined signaling interplay between SRB1 and eNOS in human umbilical vein endothelial cells (HUVECs) using RNA interference of SRB1 and inhibitor of PI3K, LY294002.

\section{Materials and Methods}

\section{- Ethical approval}

The whole experiment for cell culture involving the human tissues was approved by the affiliated hospital of Southwest Medical University (ChiCTR1800014575). Informed consent was also obtained from the participants. All procedures performed in studies involving human participants were in accordance with the ethical standards of the institutional and/or national research committee and with the 1964 Helsinki declaration and its later amendments or comparable ethical standards. This article does not contain any studies with animals performed by any of the authors.

\section{- Endothelial cell culture}

Vascular endothelial cells derived from human umbilical vein endothelial cells (HUVECs) were cultured and identified by immunohistochemistry as described previously [6]. The whole experiment for cell culture involving the human tissues was approved by the affiliated hospital of Southwest Medical University (ChiCTR1800014575). Under the aseptic conditions, fresh human umbilical cords $(15-20 \mathrm{~cm})$ were obtained from healthy women who underwent a cesarean section. Informed consent was also obtained from them. The umbilical vein was processed clean and was filled with $0.2 \%$ type II collagenase solution, subsequently digesting for $12 \mathrm{~min}$ at $37^{\circ} \mathrm{C}$. The collagenase solution was then discarded, and the vein was washed with phosphate-buffered saline (PBS) that was collected in tubes for centrifugation. After that, the supernatant was discarded, and the cell pellet was re-suspended in medium 199 (Matsa Biotechnology Co., Ltd., Shanghai, China), mixed with $1 \mathrm{~mol} / \mathrm{L}$ 4-(2-hydroxyethyl)-1-piperazineethanesulfonic acid (HEPES), 20\% fetal bovine serum (FBS; Gibco, Grand Island, NY, USA), $5 \mathrm{mg} / \mathrm{mL}$ heparin, and $100 \mu \mathrm{g} / \mathrm{mL}$ endothelial cell growth factor (Sigma, Saint Louis, MO, USA). Then the collected cells were incubated in a 5\% $\mathrm{CO}_{2}$ atmosphere at $37^{\circ} \mathrm{C}$. The culture medium was changed every other day. Once reached confluence (3 -5 days), the cells were passaged. In this study, we selected cells that grew up to the moment of the experiments. Inverted fluorescence microscopy (Olympus, CKX41, Japan) was employed to observe cell growth.

\section{Transfection of SRB1 RNA-si into HUVECS}

Three potential siRNA sequences targeting the SRB1 mRNA (the sequences are showed in TABLE 1), were designed according to the sequence recorded in NCBI, which is assisted by GenePharma Ltd (Shanghai, China). Meanwhile, a nonsense siRNA (pCMV-SRB1) was also synthesized for negative control. The endothelial cells were prepared for transfection to single out the most efficient siRNA sequence by quantitative polymerase chain reaction (q-PCR), which was also screened in the GenePharma Ltd and F1 was used in the later experiment. Subsequently, sub-confluent cells were transfected with pCMV-SRB1 or SRB1 siRNA (GenePharma Ltd, Shanghai, China) using Lipofectamine 2000 (Invitrogen, Carlsbad, CA, USA) according to the manufacturers' instructions. After transfection, endothelial cells were washed with PhosphateBuffered Saline (PBS) and incubated with Endothelial Progenitor Cell (EPC) medium for $48 \mathrm{~h}$ prior to the experiments.

\section{Administration of LY294002 inhibitor}

Before treatment of shear stress, the HUVECs were seeded onto a 6-well plate. Then cells were pretreated with LY29400 (MedChemExpress,

\begin{tabular}{|c|c|c|}
\hline \multicolumn{2}{|c|}{ TABLE 1. Information of interference fragment sequence. } \\
\hline Item & \multicolumn{1}{|c|}{ Sense (5'-3') } & Anti-sense (5'-3') \\
\hline F1 & GCGGUGAUGAUGGAGAUATT & UAUUCUCCAUCAUCACCGCTT \\
\hline F2 & GCCGAUCCAUGAAGCUAAUTT & AUUAGCUUCAUGGAUCGGCTT \\
\hline F3 & CAGGGUCCCUCAGAUUAUATT & UAUAAUCUGAGGGACCCUGTT \\
\hline
\end{tabular}

Translational Neuroscience Center, West China Hospital, Sichuan University, Chengdu 610041, China *Author for correspondence: yuluchuan@swmu.edu.cn 
Shanghai, China). After that, drug resistance was observed and analyzed. For this study, the final concentration to HUVECs that we chose was $25 \mu \mathrm{mol} / \mathrm{L}$ and it was administrated immediately before the shear stress.

\section{- Implement of shear stress}

The parallel-plate flow chamber was used to investigate the interaction of how endothelial cells are affected by shear stress (FIGURE 1). The parallel-plate flow chamber system device was described in (FIGURE 1), which was designed by Sichuan University and was manufactured by Chengdu Ximuzi Company in accordance with relevant data provided. For implement of shear stress, HUVECs were cultured in M199 media (Matsa Biotechnology Co., Ltd., Shanghai, China), mixed with $1 \mathrm{~mol} / \mathrm{L}$ HEPES, 20\% fetal bovine serum (FBS; Gibco, Grand Island, NY, USA), $5 \mathrm{mg} / \mathrm{mL}$ heparin, and $100 \mu \mathrm{g} / \mathrm{mL}$ endothelial cell growth factor (Sigma, Saint Louis, MO, USA). For shear stress, HUVECs $\left(1 \times 10^{6} / \mathrm{mL}\right)$ were seeded in $35 \times 10 \mathrm{~mm}$ glass slides, which were coated by fibronectin for 30 min in $10 \mathrm{~cm}$ culture dishes. When the cells reached the $100 \%$ confluence, they were placed into the parallel-plate flow chamber for a shear stress treatment $\left(8.4 \mathrm{dyne} / \mathrm{cm}^{2}\right)$ for $0 \mathrm{~min}, 10$ $\mathrm{min}, 30 \mathrm{~min}, 60 \mathrm{~min}$, and $120 \mathrm{~min} .0 \mathrm{~min}$ was set as the control group.

\section{- Western blotting}

To examine alternations in the protein expression levels of P-PI3K/PI3K, P-ERK/ERK, P-p38/p38, eNOs, activator protein 1 (AP-1), and $N F-\kappa B$ following $0,10,30,60$, or 120 min shear stress, or treatment of SRB1 siRNA and LY294002, protein was extracted from endothelial cells in each group and lysed with radio-immunoprecipitation assay lysis buffer (BeyotimeInstitute of Biotechnology) containing $2 \%$ inhibitor cocktail (Roche Diagnostics, Basel, Switzerland). A Bicinchoninic Acid protein assay kit (Beyotime Institute of Biotechnology) was used to detect the protein concentration. Then protein $(100 \mu \mathrm{g})$ was separated by sodium dodecyl sulfate-polyacrylamide gel electrophoresis $(15 \%)$ at $60 \mathrm{~V}$ for $30 \mathrm{~min}$ and $100 \mathrm{~V}$ for $1.5 \mathrm{~h}$. Afterwards, proteins were transferred onto polyvinylidene difluoride membranes (EMD Millipore, Billerica, MA, USA) for $4 \mathrm{~h}$ at $350 \mathrm{~mA}$, followed by blocking using 5\% skimmed milk in TBS containing Tween-20 (TBST) for $1 \mathrm{~h}$ at room temperature.

\section{The figure of flow chamber system}

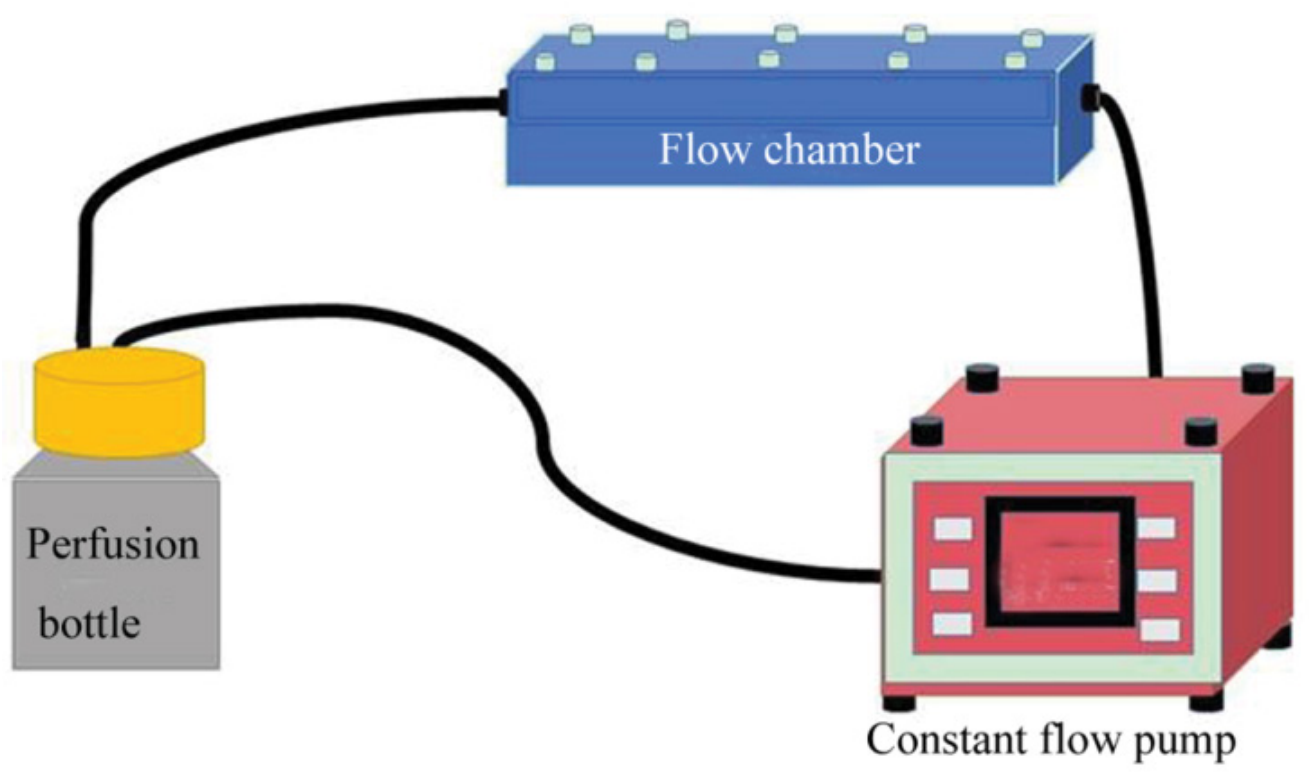

FIGURE 1. The outside view of the parallel-plate flow chamber system device. The dimensions of the flow chamber after installation are: $8.5 \mathrm{~cm}$ long, $2.5 \mathrm{~cm}$ wide, and $0.3 \mathrm{~mm}$ high. The distance between the inflow hole and the outflow hole was 7.0 $\mathrm{cm}$. The experimental device consists of a flow chamber system and a perfusion system, including a constant flow pump, a liquid storage bottle, some pipes and a flow chamber. The constant flow pump provides a steady flow for the experiment. The serumfree medium M199 is used as the perfusion liquid, and the viscosity of the perfusion liquid is $0.7651 \mathrm{mPa} \cdot \mathrm{s}$. The liquid storage bottle was maintained at 37区 in the water bath during the experiment. In the experimental process, the entire perfusion system was placed at the same plane, in order to keep hydrostatic pressure stable. 
Subsequently, the membrane was incubated with rabbit anti-rat primary antibodies against P-PI3K (rabbit anti-rat, 1:1000, SC-4228, 60 $\mathrm{kDa}$ ), PI3K (rabbit anti-rat, 1:1000, SC-4292, $85 \mathrm{kDa}$ ) P-ERK1/2 (rabbit anti-rat, 1:1000, SC9101, $42 \mathrm{kDa}$ ), ERK1/2 (rabbit anti-rat, 1:1000, SC-9102, $44 \mathrm{kDa}$ ), p38 MAPK (rabbit anti-rat, 1:200, SC-7149, $43 \mathrm{kDa}), \mathrm{P}-\mathrm{p} 38$ MAPK (rabbit anti-rat, 1:400, SC-101759, 38 $\mathrm{kDa}$ ), NF- $\mathrm{kB}$ (rabbit anti-rat, 1:100, bs-0465R, $61 \mathrm{kDa}$ ) and eNOs (Mouse, 1:500, abcam, 133 $\mathrm{kDa}$ ) as well as AP-1 (Mouse, 1:1000, abcam, $49 \mathrm{kDa}$ ) overnight at $4^{\circ} \mathrm{C}$. GAPDH (mouse, 1:500, GT239, $36 \mathrm{kDa}$ ) served as an internal control. Thereafter, the membranes were rinsed four times with TBST and incubated with Horseradish Peroxidase (HRP) conjugated goat anti-rabbit IgG secondary antibodies of Abexcel (anti-rabbit, 1:1000, Abcam) and HRP, goat anti-mouse IgG (anti-mouse, 1:5000, A21010) for 1.5 h. Finally, membranes were rinsed and detected using ChemiDoc XRS System with Image Lab Software 2.0 (Bio-Rad Laboratories,
Inc., Hercules, CA, USA) with Enhanced Chemiluminescence reagent (catalog no. BL520A; Bio-Rad Laboratories, Inc).

\section{Statistical Analysis}

All statistical analyses were analyzed using SPSS 13.0 statistical software package (SPSS Inc., Chicago, IL, USA). All data are expressed as mean \pm standard deviation (SD). Comparisons between two groups were calculated using a Student's t-test, for multiple comparisons, they were performed using one-way analysis of variance followed by Tukey's post hoc test and $P$ values $<0.05$ were considered statistically significant.

\section{Results \\ Effects of shear stress on the HUVECs and the activation of PI3K, ERK1/2, and P38}

After being treated with shear stress for 60 min, the HUVECs exhibited an irregular cell

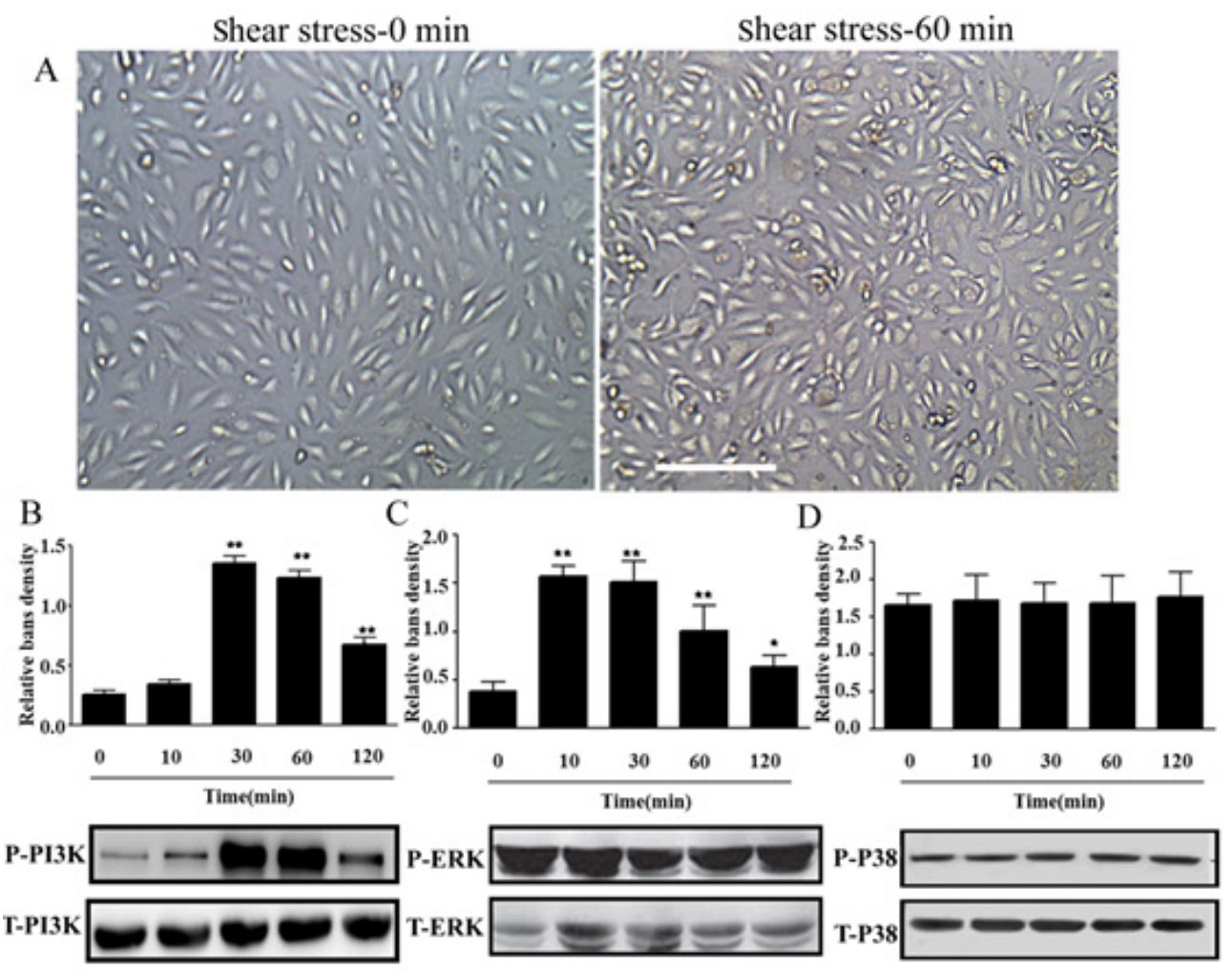

FIGURE 2. Detection of cell status and phosphorylation of PI3K, ERK1/2 and P38 under 8.4 dyne/cm ${ }^{2}$ shear stress in HUVECs for 10, 30, 60, or $120 \mathrm{~min}$. (A) The cells under 8.4 dyne $/ \mathrm{cm}^{2}$ shear stress for $0 \mathrm{~min}$ and $60 \mathrm{~min}$. Scale bar=50 $\mu \mathrm{m}$. (B) The protein expression of P-PI3K and total PI3K under 8.4 dyne/cm² for $0 \mathrm{~min}, 10 \mathrm{~min}, 30 \mathrm{~min}, 60 \mathrm{~min}$ and $120 \mathrm{~min}$. The final protein level of P-PI3K was calculated as the ratio of P-PI3K/T-PI3K. (C) The protein level of P-ERK1/2 and total ERK1/2 under $8.4 \mathrm{dyne} / \mathrm{cm}^{2}$ for 0 $\mathrm{min}, 10 \mathrm{~min}, 30 \mathrm{~min}, 60 \mathrm{~min}$ and $120 \mathrm{~min}$. The final protein level of P-ERK1/2 was calculated as the ratio of P-ERK1/2/T-ERK1/2. (D) The P-p38 and total P38 protein level under 8.4 dyne $/ \mathrm{cm}^{2}$ for $0 \mathrm{~min}, 10 \mathrm{~min}, 30 \mathrm{~min}, 60 \mathrm{~min}$ and $120 \mathrm{~min}$. The final protein level of P-p38 MAPK was calculated as the ratio of P-p38/T-p38. **P<0.01 compared with control group ( 0 min). Data are presented as the mean \pm SD. T: Total. 
morphology including increased cell gap, cell shrinkage, cells shedding, which demonstrate the action of shear stress on the HUVECs (FIGURE 2A). In addition, to clarify the signal pathways involved in shear stress-induced eNOS activation, phosphorylation of PI3K, p38 and ERK1/2 were used to assess PI3K, p38 and ERK1/2 activation, indicated by P-PI3K, P-p38, P-ERK1/2. It showed that shear stress significantly increased the protein level of P-PI3K, which was calculated by the ratio of P- PI3K / total PI3K (T-PI3K) as the final level after exposure for $30 \mathrm{~min}$ and lasting for at least $120 \mathrm{~min}$ (FIGURE 2B, $P<0.05$ ). In contrast, there was no effect on the protein level of P-p38, P-ERK1/2 (FIGURE 2C and D, P>0.05). Basically, we got that shear stress can up-regulate P-PI3K expression in the HUVECs.

\section{SRB1 interference could inhibit the shear stress-induced PI3K activation}

To verify that shear stress induced eNOS activation via SRB1, we used RNA interference (siRNA) to inhibit SRB1, then protein level of P-PI3K/PI3K was examined by western blot. As shown in FIGURE 3, compared with the control group, shear stress induced increased protein level of P-PI3K, while this was partly reversed by SRB1 siRNA transfection as compared with shear stress group, but it was still higher than that in the control group (FIGURE 3, $P<0.05$ ). These indicate that SRB1 interference could inhibit the activation of PI3K after shear stress.

\section{Administration of LY294002 could block the shear stress-induced eNOS activation}

To further verify whether PI3K was involved in regulating eNOS expression following shear stress, the inhibitor was used to block the PI3K pathway. The results revealed that compared with the control group, the eNOS protein expression was significantly increased under 8.4 dyne $/ \mathrm{cm}^{2}$ shear stress, while it was significantly decreased when the PI3K was blocked by LY294002 inhibitor, which was still higher than that in the control group (FIGURE 4, $P<0.05$ ).

\section{Inhibition of SRB1 and PI3K could reduce the DNA binding activity of AP-1 and NF-KB under the shear stress}

The binding activity of nuclear transcription factor AP-1 and DNA at different time points under 8.4 dyne $/ \mathrm{cm}^{2}$ shear stress was further

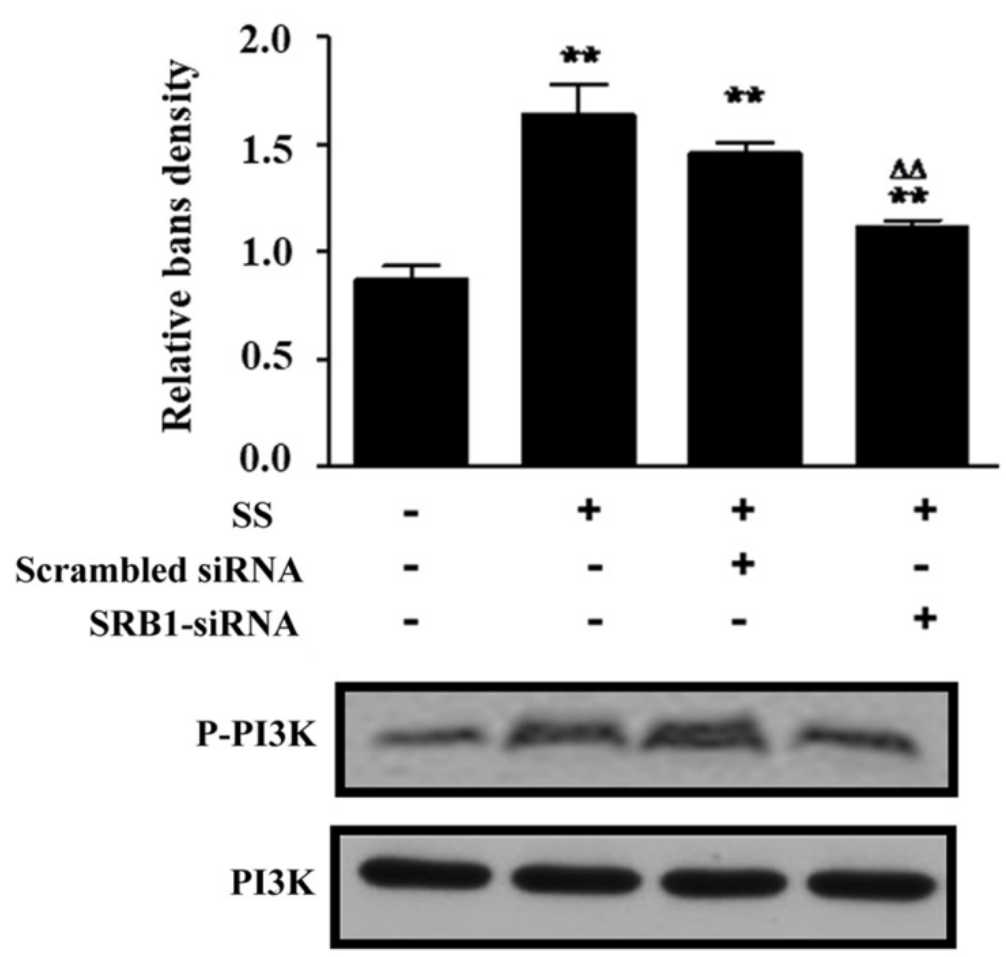

FIGURE 3. Effect of SRB1 on PI3K activation after shear stress. The four columns were control, Shear stress, Shear stress+Scrambled siRNA and Shear stress+SR-B1 siRNA groups, respectively; ${ }^{* *}$ compared with control group, $p<0.01$; ${ }^{*}$ CSompared with control group, $\mathrm{p}<0.05 ;{ }^{\triangle} \Delta$ compared with shear stress group, $\mathrm{p}<0.01$. SS; Shear stress. The final protein level of $\mathrm{P}-\mathrm{PI} \mathrm{K}$ was calculated as the ratio of P-PI3K/T-PI3K. 

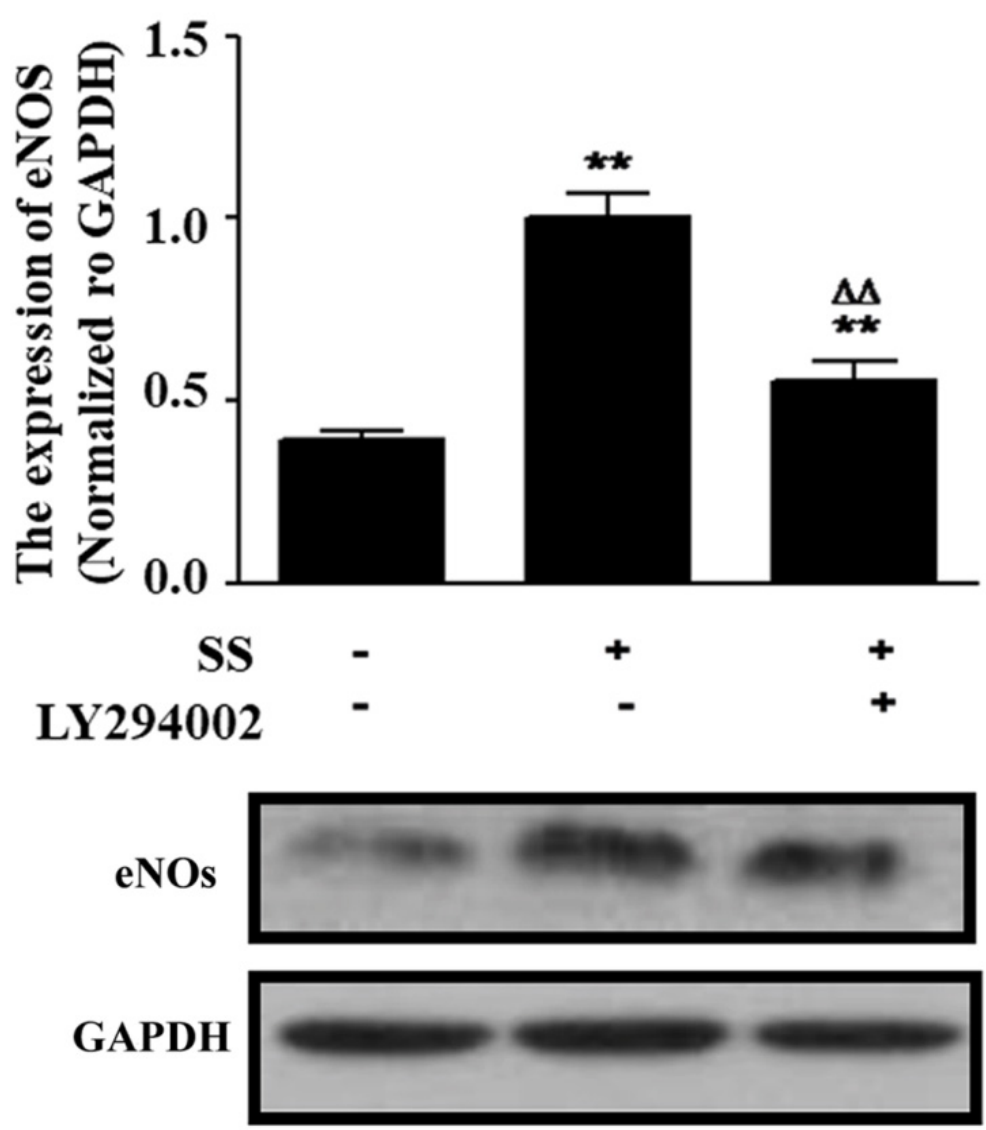

FIGURE 4. Effect of PI3K inhibition on the eNOS activity after shear stress. The protein expression level of eNOS protein. The three columns were control, Shear stress, and Shear stress+LY294002 groups, respectively; ${ }^{* *}$ Compared with control group, $\mathrm{p}<0.01 ;{ }^{\triangle \triangle}$ Compared with shear stress group, $\mathrm{p}<0.01 . \mathrm{SS} ;$ Shear stress.

studied. The results showed that for AP-1, binding activity was increased from 10 to 120 min (FIGURE 5A, $P<0.05$ ), however, for NF$\kappa \mathrm{B}$, binding activity was only increased at 30 and $60 \mathrm{~min}$ (FIGURE 5B, $P<0.05$ ). To confirm whether this effect was mediated by SRB1and PI3K, the LY294002 and siRNA-SRB1 were used to inhibit PI3K and SRB1, respectively. As expected, AP-1 DNA binding activity was significantly decreased by treatment with LY294002 inhibitor or SRB1 siRNA, when compared with the shear stress control group (FIGURE 5C, $P<0.05$ ). However, there was no effect on NF- $\kappa \mathrm{B}$ binding (FIGURE 5D, $P<0.05)$.

\section{Discussion}

AS is a complicated chronic process that involves artery wall inflammation, endothelial cell dysfunction, and altered hemodynamic forces [14]. Some studies have shown that shear stress can be transduced into intracellular signals in vascular endothelial cells, ultimately resulting in eNOS activation and Nitric Oxide (NO) production [15-17]. Several studies have been carried out to identify some proteins that could act as a mechanosensor $[18,19]$. In this process, our previous work showed that SRB1 is such a mechanosensor expressed on the endothelial membrane that can convert mechanical signals into chemical signals and activate eNOS, however, the underlying mechanism of SRB1 in this process remains unclear. The present study was performed to determine whether P38, ERK1/2 or PI3K are involved in this signaling pathway.

In this study, firstly, we found that the PI3K phosphorylation was increased after shear stress exposure for $30 \mathrm{~min}$ and lasting for at least 120 min and shear stress did not affect P38 and ERK1/2 phosphorylation, identified PI3K as a critical regulator in shear stress-induced eNOS activation. Compared with P38 and ERK1/2, many previous studies have mentioned that among proteins involved in shear stressinduced induced eNOS phosphorylation [20] 

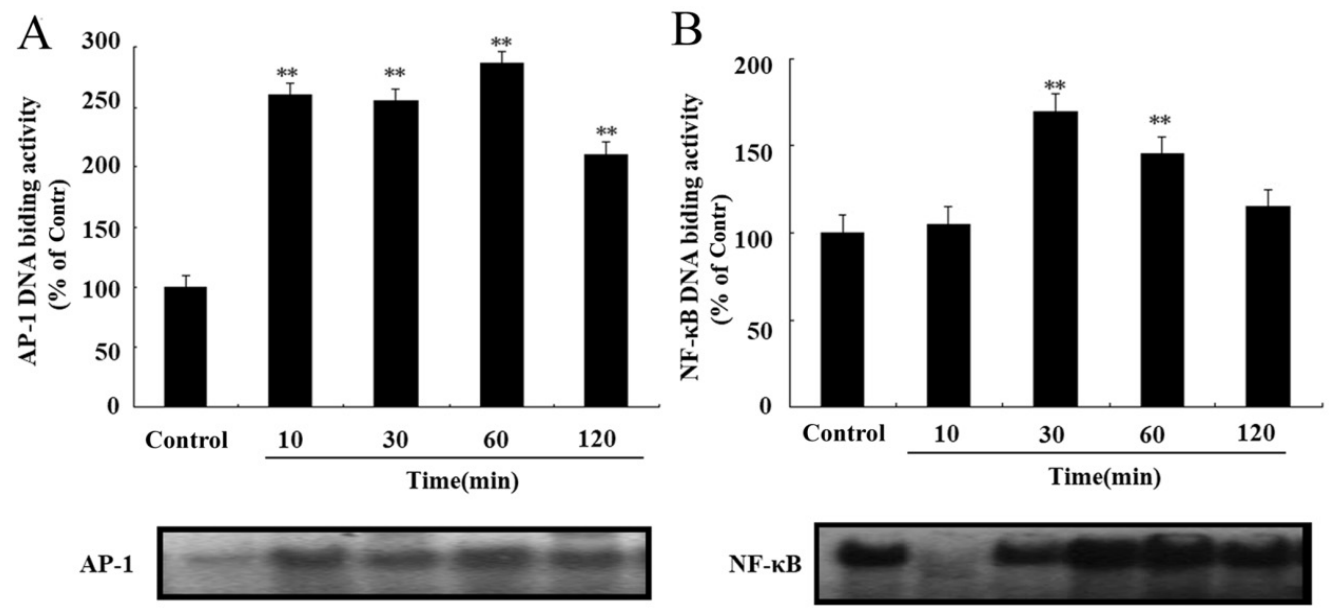

$\mathrm{C}$

$\mathrm{D}$
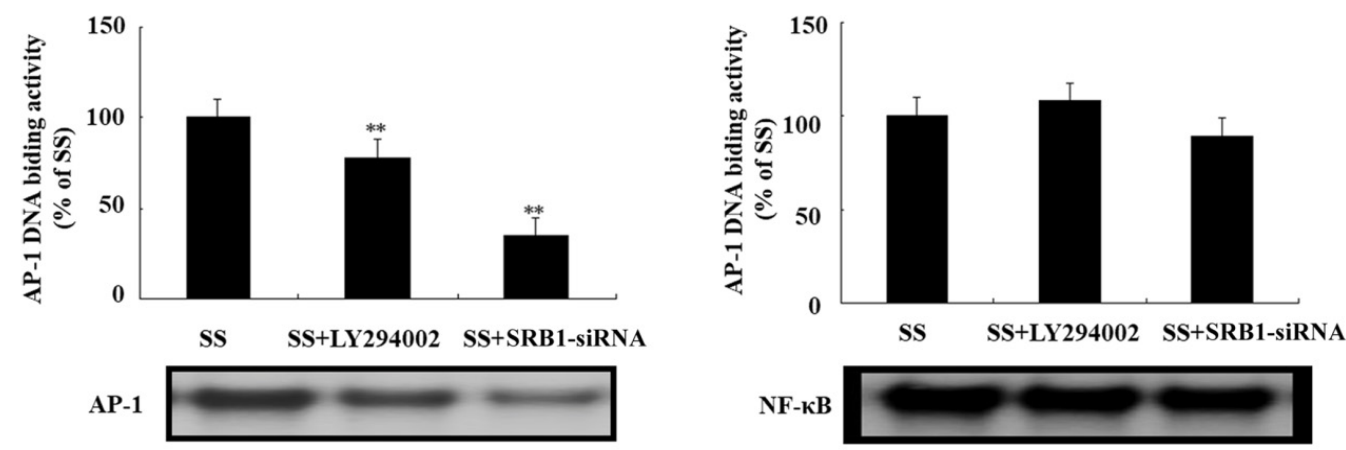

FIGURE 5. Effects of SRB1 and PI3K on the DNA binding activity of AP-1 and NF-KB under shear stress. (A) AP-1 DNA binding activity under $8.4 \mathrm{dyne} / \mathrm{cm}^{2}$ for $0 \mathrm{~min}, 10 \mathrm{~min}, 30 \mathrm{~min}, 60 \mathrm{~min}$ and $120 \mathrm{~min}$. The final protein level of AP-1 was calculated as the ratio of control group. (B) NF-KB DNA binding activity under 8.4 dyne/cm² for $0 \mathrm{~min}, 10 \mathrm{~min}, 30 \mathrm{~min}, 60 \mathrm{~min}$ and $120 \mathrm{~min}$. The final protein level of NF-KB was calculated as the ratio of control group. ${ }^{*}$ Compared with control group, $\mathrm{P}<0.01$. (C) AP-1 and DNA binding activity under Shear stress, Shear stress+LY294002 and Shear stress+SRB1-siRNA, respectively. The final protein level of AP-1 was calculated as the ratio of SS group. (D) NF-KB DNA binding activity under Shear stress, Shear stress+LY294002 and Shear stress+SRB1-siRNA, respectively. The final protein level of NF-KB was calculated as the ratio of SS group. ${ }^{* *}$ Compared with control shear stress, $\mathrm{P}<0.01$. SS; Shear stress.

and oxidative damage [21,22], only PI3K could be regulated by shear stress; Notably, the PI3K inhibitor LY294002 could block this effect. Then, to test whether shear stressregulated eNOS via SRB1-PI3K signaling, we inhibited SRB1 and PI3K with SRB1 RNAi and LY294002, respectively. Under these conditions, when SRB1 was blocked, PI3K phosphorylation could be inhibited and when PI3K was inhibited, eNOS expression was significantly decreased. This suggests that in endothelial cells, external shear stress is transduced into the cytoplasm by SRB1, and this signal may transduce into the nucleus via the PI3K pathway.

SRB1 is abundantly expressed in almost all endothelial cells [23] and is a key player in HDL metabolism. The extracellular loop of SRB1 can interact with a variety of lipoproteins including HDL [24]. Extrahepatic production of SRB1 has been shown to be athero-protective, but it is not known which cells are involved [25]. Expression of SRB1 in endothelial cells has been shown to be beneficial for HDL activation of eNOS [26]. We previously reported that shear stress could regulate SRB1 expression in endothelial cells and activate eNOS $[6,13]$. Recent studies indicated that increased SRB1 expression in endothelial cells could modulate lipid and lipoprotein levels and protect against AS in mice [27,28]. Based on the above, we can infer that 8.4 dyne $/ \mathrm{cm}^{2}$ shear stress could protect against AS through modulating lipid and lipoprotein levels and activating eNOS.

With regard to signaling pathways involved, one group found that HDL enhanced transendothelial cholesterol transport by activating a mechanism involving ABCA1, ABCG1, and SRB1 but not involving PI3K or Akt [29]. 
Another study reported that SRB1-mediated PI3K-Akt-eNOS signaling was involved in HDL-induced cyclo-oxygenase 2 expression and prostacyclin release in endothelial cells [30]. Moreover, low macrophage expression of SRBI promoted defective efferocytosis signaling via the Src/PI3K/Rac1 pathway, resulting in increased plaque size, necrosis, and inflammation [31].

Based on other nuclear signaling studies [32-34], we studied the DNA binding activity of AP-1 and NF- $\kappa$ B and found that AP-1 was regulated by shear stress. Importantly, this effect could be attenuated by SRB1 siRNA and LY294002. Our findings suggest that nuclear factor AP-1 is also the key regulator that transduces hydrodynamic signals into the nucleus to affect protein expression.

In summary, SRB1 plays a crucial role in mediating shear stress-induced eNOS activation that controls endothelial cell function. In the cytoplasm, PI3K is the key mediator of mechanical signal transduction, while AP-1 plays this role in the nucleus. The signaling pathways that mediate the prevention of AS include SRB1-PI3K/AP1-eNOS. This pathway is likely to be a great significance for AS prevention and treatment.

\section{Funding}

This experiment was funded by Sichuan Science and Technology Department-Luzhou Government-Luzhou Medical College cooperation project (14JC0173); Collaborative Innovation Center for Prevention and Treatment of Cardiovascular Disease of Sichuan science and technology project (2018JY0080).

\section{Availability of Data and Materials}

The analyzed datasets generated during the study are available from the corresponding author on reasonable request.

\section{Authors' Contributions}

YZ, YHD, and FXY made substantial contributions to conception and design analysis as well as interpretation of data; YZ, YHD, LLX, JYW, YMN and XJX involved in acquisition of data and assessment of study quality; YZ and YHD performed the manuscript draft; YF and BL revised manuscript scrupulously for important intellectual content; All authors finally approved the version that would be released.

\section{Compliance with Ethical Standards - Disclosure of potential conflicts of interest}

The authors declare that there is no conflict of interest regarding the publication of this paper.

\section{Ethics approval and consent to participate}

The whole experiment for cell culture involving the human tissues was approved by the affiliated hospital of Southwest Medical University (ChiCTR1800014575). Informed consent was also obtained from the participants. All procedures performed in studies involving human participants were in accordance with the ethical standards of the institutional and/or national research committee and with the 1964 Helsinki declaration and its later amendments or comparable ethical standards.

\section{- Patient consent for publication}

Not applicable

\section{Acknowledgments}

Not applicable 


\section{References}

Davies PF, Dewey CF, Bussolari SR, et al. Influence of hemodynamic forces on vascular endothelial function. In vitro studies of shear stress and pinocytosis in bovine aortic cells. J. Clin. Invest. 73(4), 1121-1129 (1984).

Haining AW, Lieberthal TJ, Del Río HA. Talin: a mechanosensitive molecule in health and disease. FASEB J. 30(4), 2073-2085 (2016).

Maimari N, Pedrigi RM, Russo A, et al. Integration of flow studies for robust selection of mechanoresponsive genes. Thromb. Haemost. 115(3), 474-483 (2016).

Kwak BR, Back M, Bochaton-Piallat ML, et al. Biomechanical factors in atherosclerosis: mechanisms and clinical implications. Eur. Heart J. 35(43), 30133020 (2014).

Passerini AG, Polacek DC, Shi C, et al. Coexisting proinflammatory and antioxidative endothelial transcription profiles in a disturbed flow region of the adult porcine aorta. Proc. Natl. Acad. Sci. USA. 101(8), 2482-2487 (2004).

Yu F, Zhang Y, Ling S, et al. The study of the influence of different shear stress on the mRNA expression of scavenger receptor class $B$ type 1 in endothelial cells. Sheng Wu Yi Xue Gong Cheng Xue Za Zhi. 28(1), 81-85 (2011).

Yuhanna IS, Zhu Y, Cox BE, et al. High-density lipoprotein binding to scavenger receptor-BI activates endothelial nitric oxide synthase. Nat. sMed. 7(7), 853-857 (2011).

Chiu JJ, Chien S. Effects of disturbed flow on vascular endothelium: pathophysiological basis and clinical perspectives. Physiol. Rev. 91(1), 327-387 (2011).

Mineo C, Shaul PW. Functions of scavenger receptor class $\mathrm{B}$, type $\mathrm{I}$ in atherosclerosis. Curr. Opin. Lipidol. 23(5), 487-493 (2012).

Nigro P, Abe J, Berk BC. Flow shear stress and atherosclerosis: a matter of site specificity. Antioxid. Redox. Signal. 15, 1405-1414 (2011).
Gao M, Zhao D, Schouteden S, et al. Regulation of high-density lipoprotein on hematopoietic stem/progenitor cells in atherosclerosis requires scavenger receptor type BI expression. Arterioscler. Thromb. Vasc. Biol. 34, 1900-1909 (2014).

Ashraf MZ, Gupta N. Scavenger receptors:implicationsinatherothrombotic disorders. Int. J. Biochem. Cell. Biol. 43(5), 697-600 (2011).

Zhang Y, Liao B, Li M, et al. Shear stress regulates endothelial cell function through SRB1-eNOS signaling pathway. Cardiovasc. Ther. 34(5), 308-313 (2016).

Zaragoza C, Marquez S, Saura M. Endothelial mechanosensors of shear stress as regulators of atherogenesis. Curr. Opin. Lipidol. 23(5), 446-452 (2012).

Huang C, Bruggeman LA, Hydo LM, et al. Shear stress induces cell apoptosis via a c-Src-phospholipase D-mTOR signaling pathway in cultured podocytes. Exp. Cell Res. 318(10), 1075-1085 (2012).

Sriram K, Laughlin JG, Rangamani $P$, et al. Shear-induced nitric oxide production by endothelial cells. Biophys. $J$. 111(1), 208-221 (2016).

Kemeny SF, Figueroa DS, Clyne AM. Hypo-and hyperglycemia impairs endothelial cell actin alignment and nitric oxide synthase activation in response to shear stress. PLoS One. 8(6), (2013).

Coon BG, Baeyens N, Han J, et al. Intramembrane binding of $\mathrm{VE}$-cadherin to VEGFR2 and VEGFR3 assembles the endothelial mechanosensory complex. $J$. Cell Biol. 208(7), 975-986 (2015).

Rampersad SN, Wudwud A, Hubert F, et al. Adaptive phenotypic modulation of human arterial endothelial cells to fluid shear stress-encoded signals: modulation by phosphodiesterase 4D-VE-cadherin signaling. Cell Signal. 28(7), 741-748 (2016).

Bretón RR, González de OC, Romero $\mathrm{N}$, et al. Critical role of hydrogen peroxide signaling in the sequential activation of p38 MAPK and eNOS in laminar shear stress. Free Radic. Biol. Med. 52(6), 10931100 (2012).

Wang Z, Zhang J, Li B, et al. MAPK signaling mediates low shear stress-induced oxidative damage in human umbilical vein endothelial cells in vitro. Nan Fang $\mathrm{Yi}$ Ke Da Xue Xue Bao. 34 (5), 603-608 (2014).

Huang A, Yang YM, Yan C, et al. Altered MAPK signaling in progressive deterioration of endothelial function in diabetic mice. Diabetes. 61(12), 31813188 (2012).

Bin G, Bo Z, Jing W, Jin J, et al. Fluid shear stress suppresses TNF- $₫$ induced apoptosis in MC3T3-E1 cells: Involvement of ERK5-Akt-FoxO3a-Bim/ FasL signaling pathways. Exp. Cell Res. 343(2), 208-217 (2016).

Mineo C, Shaul PW. Functions of scavenger receptor class $\mathrm{B}$, type I in atherosclerosis. Curr. Opin. Lipidol. 23(5), 487-493 (2012).

Huby T, Doucet C, Dachet C, et al. Knockdown expression and hepatic deficiency reveal an atheroprotective role for SR-BI in the liver and peripheral tissues. J. Clin. Invest. 116(10), 27672776 (2006).

McAllister RM, Morris DM, Weimer $\mathrm{CM}$, et al. Effects of high-density lipoprotein on endothelium-dependent vasorelaxation. Appl. Physiol. Nutr. Metab. 35(3), 319-327 (2010).

Vaisman BL, Vishnyakova TG, Freeman LA, et al. (2015) Endothelial expression of scavenger receptor class $\mathrm{B}$, Type I protects against the development of atherosclerosis in mice. Biomed. Res. Int. 1, (2015).

Armstrong SM, Sugiyama MG, Fung KY, et al. A novel assay uncovers an unexpected role for SR-BI in LDL transcytosis. Cardiovasc. Res. 108(2), 268277 (2015).

Miao L, Okoro EU, Cao Z, et al. Highdensity lipoprotein-mediated transcellular cholesterol transport in mouse aortic endothelial cells. Biochem. Biophys. Res. Commun. 465 (2), 256-261 (2015).

Zhang QH, Zu XY, Cao RX, et al. An involvement of SRB1 mediated PI3K-Akt-eNOS signaling in HDLinduced cyclooxygenase 2 expression and prostacyclin production in endothelial cells. Biochem. Biophys. Res. Commun. 
420(1), 17-23 (2012).

Tao H, Yancey PG, Babaev VR, et al. Macrophage SR-BI mediates efferocytosis via Src/PI3K/Rac1 signaling and reduces atherosclerotic lesion necrosis. J. Lipid. Res. 56(8), 1449-1460 (2015).

Luff SA, Papoutsakis ET. Megakaryocytic maturation in response to shear flow is mediated by the activator protein 1 (AP-1) Transcription factor via Mitogen-Activated Protein Kinase (MAPK) Mechanotransduction. J. Biol. Chem. 291(15), 7831-7843 (2016).

Rohrer L, Ohnsorg PM, Lehner M, et al. High-density lipoprotein transport through aortic endothelial cells involves scavenger receptor BI and ATP-binding cassette transporter G1. Circ. Res. 104(10),
1142-1150 (2009).

Lin X, Yang $\mathrm{H}$, Zhang $\mathrm{H}$, et al. A novel transcription mechanism activated by ethanol: induction of Slc7a11 gene expression via inhibition of the DNAbinding activity of transcriptional repressor octamer-binding transcription factor 1 (OCT-1). J. Biol. Chem. 288(21), 14815-14823 (2013). 